\title{
Comunicación
}

\section{ESTUDIO PRELIMINAR DE COLECCIÓN DE SEMEN EN OSO DE ANTEOJOS (Tremarctos ornatus)}

\author{
Marco Enciso H. ${ }^{1,2}$, Lizette Bermúdez L. ${ }^{3}$, Shirley Evangelista V. ${ }^{1}$, \\ Gianmarco Rojas M. ${ }^{3}$ y Wilfredo Huanca L. ${ }^{4}$
}

\section{Abstract}

\begin{abstract}
Semen was collected in a Spectacled Bear (Tremarctos ornatus) reared in captivity using the electroejaculation technique. Four series of 6 volt discharges by 15 seconds each plus manual stimulation were carried out. An effective penis erection and small volume of ejaculate was obtained in the last series of electrical stimulus. Seminal motility was $50 \%$. Further studies are required to optimize the use of the electroejaculator in order to obtain higher volumes and better semen quality.
\end{abstract}

Key words: semen collection, electroejaculation, spectacled bear, Tremarctos ornatus

El oso de anteojos u oso andino (Tremarctos ornatus), único representante de la familia Ursidae que habita en América del Sur, se distribuye desde Venezuela hasta Bolivia y en diversos hábitats y altitudes (Figueroa y Stucchi, 2003). El Perú es el país con mayor densidad poblacional de osos de anteojos, teniendo un aproximado de 2,000 a 6,000 individuos (Peyton, 1999). Se le considera una "especie símbolo" en el ámbito de la conservación, debido al rol que cumple dentro del ecosistema y su influencia sobre el poblador andino y su cultura (Peyton, 1999).
Esta especie está considerada como vulnerable por la Unión Internacional para la Conservación de la Naturaleza (IUCN), y está categorizada por la Convención Sobre el Comercio Internacional de Especies Amenazadas de Fauna y Flora Silvestres (CITES) dentro del Apéndice I. En forma similar, el Instituto Nacional de Recursos Naturales (INRENA) la considera como especie en peligro encontrándose protegida mediante el D.S. 013-99-AG (Figueroa y Stucchi, 2003). Las poblaciones de osos de anteojos han sufrido una drástica disminución en las últimas décadas debido a la cacería

\footnotetext{
${ }^{I}$ Práctica privada

${ }^{2}$ E-mail: marco.enciso@gmail.com

${ }^{3}$ Parque Zoológico Huachipa

${ }^{4}$ Laboratorio de Reproducción Animal, FMV-UNMSM
} 
indiscriminada, ya sea por deporte, por considerarlo como una especie dañina para la actividad ganadera y agrícola, por creencias místico-religiosas del poblador andino y por la paulatina destrucción de su hábitat (Figueroa, 2003).

La reproducción artificial o asistida es una alternativa que debe ser considerada para paliar el problema poblacional del oso de anteojos. Para esto, se requiere desarrollar protocolos de colección de semen entre otros aspectos. Se dispone de diversas técnicas en animales domésticos para dicho fin, pero en el caso de esta especie el método de electroeyaculación parece ser un procedimiento apropiado, como ha sido corroborado en diferentes especies silvestres (Durrant, 1990; Howard, 1993). Esta técnica se basa en la respuesta de erección-eyaculación como producto de estímulos eléctricos aplicados a través de un transductor por vía rectal, que activa el eje del sistema nervioso relacionado con el tracto reproductivo (Herrick y Self, 1965; Howard, 1993).

Es así, que se planteó un estudio para evaluar la técnica de electroeyaculación en el oso de anteojos para la colección de semen. Se utilizó un ejemplar de oso de anteojos adulto del Parque Zoológico Huachipa de Lima, Perú. El animal fue inmovilizado químicamente e inducido a un plano anestésico quirúrgico administrándole ketamina $(8 \mathrm{mg} /$ $\mathrm{kg})$, xilacina $(1 \mathrm{mg} / \mathrm{kg})$ y atropina $(0.04 \mathrm{mg} /$ $\mathrm{kg}$ ), adaptando lo descrito por Caulkett y Cattet (2002). El estado de anestesia se mantuvo mediante la administración inhalatoria de isofluorano al 3\%. Para la colección de semen, el animal fue colocado en posición decúbito dorsal, se rasuró la zona prepucial, la misma que se lavó con una solución de $\mathrm{ClNa}$ al $0.9 \%$ y agua tibia, para luego exponer el pene (Fig. 1).

Se empleó un electroeyaculador portátil diseñado para ovinos y caprinos (Bailey Ejaculator®, Western Instrument Company) de 6v (Fig. 2). El transductor se introdujo 15 $\mathrm{cm}$ por vía rectal con ayuda de un gel lubricante.

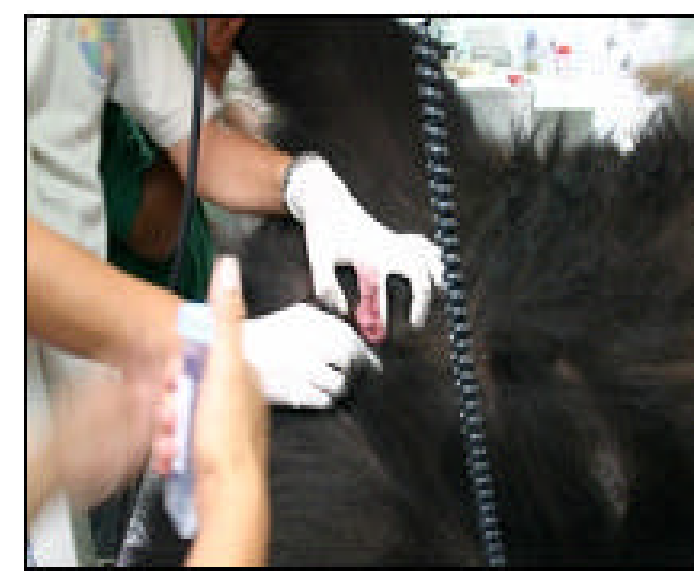

Figura 1. Exposición del pene del oso de anteojos previo a la electroeyaculación

Se aplicaron descargas eléctricas similares a las que son aplicadas en animales domésticos (Herrick y Self, 1965). El procedimiento consistió en cuatro series consecutivas, con intervalos de 2 minutos entre la $1^{\text {a y }}$ $2^{\mathrm{a}}$ y entre la $2^{\mathrm{a}}$ y $3^{\mathrm{a}}$ serie, y de 10 min entre la

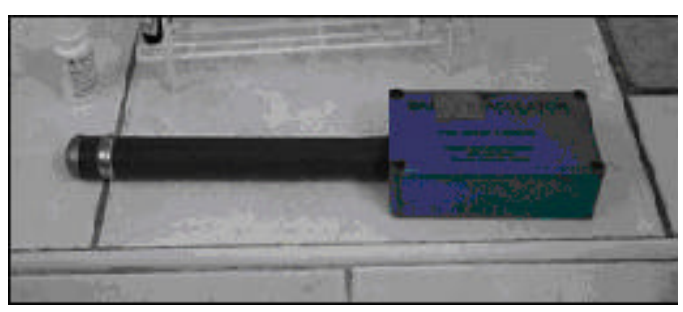

Figura 2. Electroeyaculador usado para la colección de semen en el oso de anteojos

$3^{\mathrm{a}}$ y $4^{\mathrm{a}}$ serie. En todas las series se aplicaron secuencialmente estímulos de 6, 5 y 4 segundos con descansos de 5 segundos para la $1^{\mathrm{a}}$ serie, de 3 segundos para la $2^{\mathrm{a}}$ y $3^{\mathrm{a}}$ serie y de 2 segundos para la $4^{\mathrm{a}}$ serie. En todo el proceso se adicionó una estimulación manual.

Se pudo obtener una erección efectiva a partir de la última serie de descargas, con eyaculación de una pequeña cantidad de semen que fue evaluada directamente según lo descrito por Ax et al. (2002). Se observó la 


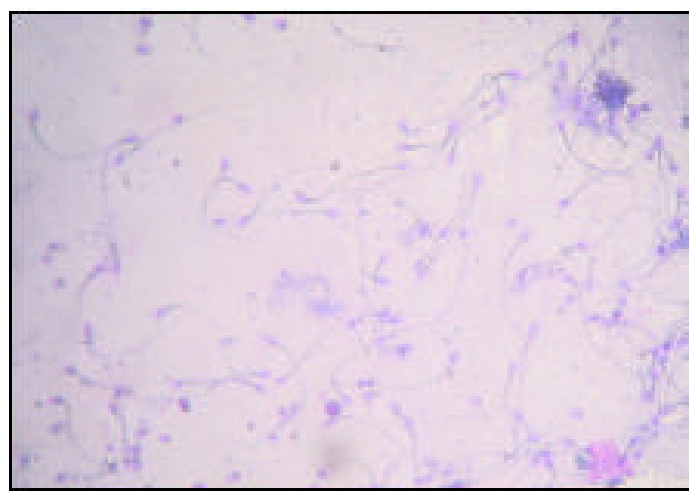

Figura 3. Espermatozoides de oso de anteojos (Tremarctos ornatus). Tinción Diff Quick. 40x

presencia de células espermáticas con una motilidad progresiva del $50 \%$. La lámina de semen fue fijada en alcohol de $70^{\circ}$ y teñida con coloración Diff Quick (Tinción 15®, Biopur, Rosario, Argentina) (Fig. 3).

Este es el primer reporte de colección de semen en el Oso de Anteojos (Tremarctos ornatos) en el país. Si bien se pudo colectar una muestra significativa de semen con el método de la electroeyaculación, la cantidad fue insuficiente para una evaluación completa de semen. La respuesta al electroeyaculador varía entre especies e individuos, y esto puede depender del tipo de equipo (voltaje, tamaño del transductor, etc), del tiempo utilizado en y entre descargas, de allí que haya que realizar estudios adicionales.

El 50\% de motilidad puede ser considerado como bajo y no necesariamente tiene que ser atribuido al animal, sino también al protocolo de colección utilizado. Como referencia se dispone de los reportes para Panda Gigante (Ailuropoda melanoleuca) con motilidad del 67.5\% (Platz et al., 1983), del Oso Pardo Japonés (Ursus arctos yesoensis) con 80\% (Ishikawa et al., 1998) y del Oso Negro Asiático (Ursus thibetanus japonicus) con $73 \%$ (Kojima et al., 2002).

\section{Literatura Citada}

1. Ax, R.L.; M. Dally; B.A. Didion; R.W. Lenz; C.C. Love; D.D. Varner; B. Hafez; M.E. Bellin. 2002. Evaluación del semen. En: Reproducción e inseminación artificial en animales. Hafez, E.S.E.; B. Hafez (eds). 7 ed. p 375386. Ed. McGraw-Hill Interamericana. México DF.

2. Caulkett, N.; M.R.L. Cattet. 2002. Anesthesia of bears. En: Zoological restraint and anesthesia. Heard, D. (ed). International Veterinary Information Service (IVIS), Ithaca, New York, U.S.A. Disponible en: http:// www.ivis.org/special books/Heard/ caulkett/IVIS.pdf

3. Durrant, B. 1990. Semen collection, evaluation, and cryopreservation in exotic animal species: Maximizing reproductive potential. ILAR J. 32: 2-10.

4. Figueroa, J. 2003. Cacería del oso andino en el Perú: Etnozoología y comercio. Cooperación Técnica AlemanaGTZ/FANPE. Proyecto Oso Andino Perú. Lima-Perú. 66 p.

5. Figueroa, J.; M. Stucchi. 2003. Algunos alcances sobre la situación del Oso Andino Tremarctos ornatus (Cuvier, 1825) en cautiverio en el Perú. XI Congreso de la Asociación Latinoamericana de Parques Zoológicos, Acuarios y Afines. Lima. 6 p.

6. Herrick, J.; H. Self. 1965. Evaluación de la fertilidad del toro y del verraco. $p$ 19-43. Ed. Acribia. Zaragoza, España.

7. Howard, J.G. 1993. Semen collection and analysis in carnivores. En: Zoo \& Wild Animal Medicine. Current Therapy 3. Fowler, M.E. (ed). $3^{\text {rd }}$ ed. p 390-399. W.B. Saunders. USA.

8. Ishikawa, A.; M. Matsui; H. Tsuruga; H. Sakamoto; Y. Takahashi; H. Kanagawa. 1998. Electroejaculation and semen characteristics of the captive Hokkaido Brown Bear (Ursus arctos yesoensis). J. Vet. Med. Sci. 60: 965-968. 
9. Kojima, E.; H. Tsuruga; T. Komatsu; T. Murase; T. Tsubota; I. Kita. 2001. Characterization of semen collected from Beagles and captive Japanese Black Bears (Ursus thibetanus japonicus). Theriogenology 55: 717-731.

10. Peyton, B. 1999. Spectacled bear conservation action plan. En: Bears. Status survey and conservation action plan.
Servheen, C.; S. Herrero; B. Peyton. (eds). p 157-198. IUCN/SCC Bear Specialist Group. Switzerland and Cambridge. UK.

11. Platz, C.C. Jr.; D.E. Wildt; J.G. Howard; M. Bush. 1983. Electroejaculation and semen analysis and freezing in the Giant Panda (Ailuropoda melanoleuca). J. Reprod. Fert. 67: 9-12. 